East African Medical Journal Vol. 86 No. 9 September 2009

NEPHROTOXICITY OF AMPHOTERICINB INTHE TREATMENT OF CRYPTOCOCCALMENINGITIS INACQUIRED IMMUNODEFICIENCY SYNDROME PATIENTS

P. O. Ochieng, MBChB, P. O. Box 58979 - 00200, Nairobi, Kenya, S. O. McLigeyo, MBChB, MMed (Nbi), Associate Professor, E. O. Amayo, MBChB, MMed (Nbi), Associate Professor, J. K. Kayima, MBChB, MMed (Nbi), Senior Lecturer, and E. O. Omonge, MBChB, MMed (Nbi), Department of Clinical Medicine and Therapeutics, College of Health Sciences, University of Nairobi, P. O. Box 19676-00202, Nairobi, Kenya

Request for reprints to: Dr. P. O. Ochieng, P. O. Box 58979-00200, Nairobi, Kenya

\title{
NEPHROTOXICITY OF AMPHOTERICIN B IN THE TREATMENT OF CRYPTOCOCCAL MENINGITIS IN ACQUIRED IMMUNODEFICIENCY SYNDROME PATIENTS
}

\author{
P. O. OCHIENG, S. O. McLIGEYO, E. O. AMAYO, J. K. KAYIMA and E. O. OMONGE
}

\begin{abstract}
Objectives: To describe the incidence of renal dysfunction, hypokalaemia and hypomagnesaemia in AIDS patients with cryptococcal meningitis and on amphotericin B treatment. Secondary objective was to determine all-cause mortality in the same group. Design: Prospective, observational study.

Setting: Kenyatta National Hospital (KNH), Nairobi, Kenya.

Subjects: Seventy consecutive patients with AIDS and cryptococcal meningitis on amphotericin B.

Results: About $58.6 \%$ of the patients had at least $100 \%$ rise in the creatinine level. Thirty eight point six per cent of patients experienced a rise in serum creatinine of at least $50 \%$. Ninty three per cent of the patients developed hypokalaemia and $80 \%$ had hypomagnesaemia at trough magnesium level. Only $54.3 \%$ of patients completed the intended 14-day treatment. Thirty point five per cent of patients died within the two week follow-up period.

Conclussion: The incidences of amphotericin B associated nephrotoxicity, hypokalemia and hypomagnesaemia were high in this studied population.
\end{abstract}

\section{INTRODUCTION}

Sub-Saharan Africa accounts for about $68 \%$ of the reported 33.2 million HIV / AIDS cases in 2007 (1). Kenya contributes its share to this pandemic with an estimated $7 \%$ prevalence rate (2). Cryptococcal meningitis is a common opportunistic infection in AIDS patients, particularly in Southeast Asia and Africa $(3,4)$ and it is associated with high mortality (5).

Amphotericin B is the drug of choice for the treatment of severe systemic fungal illnesses, including cryptococcal meningitis. However, the main deterrent for its use is nephrotoxicity. Impaired renal function is a common complication of using amphotericin B, occurring in 5 to $80 \%$ of cases (610). Renal dysfunction includes: renal insufficiency, urinary potassium wasting and hypokalaemia., urinary magnesium wasting and hypomagnesaemia., metabolic acidosis due to type 1 (or distal) renal tubular acidosis, and polyuria due to nephrogenic diabetes insipidus $(6-8,11)$.
Pharmaco-economic consideration leaves amphotericin B deoxycholate as the drug of choice for treatment of cryptococcal meningitis in Kenya and other developing nations. Nephrotoxicity of amphotericin B adversely affects mortality, length of hospital stay and cost of treatment (12-14).

Nephrotoxicity associated with amphotericin B is usually reversible with discontinuation of therapy but can recur if treatment is reinstituted $(15,16)$.

There is no universal consensus on monitoring of amphotericin B therapy but most commonly used tests include serum urea and serum creatinine concentration, complete blood count $(\mathrm{CBC})$, serum magnesium and potassium levels.

The main objective of this study was to determine the incidence of nephrotoxicity through serial creatinine, creatinine clearance, potassium and magnesium measurements. These were correlated with demographic variables. The secondary objectives were to determine the two week all cause mortality and the proportion of patients failing to complete the intended 14-day therapy. 


\section{MATERIALS AND METHODS}

Between July and October 2007, 84 patients with AIDS aged 18 years and above, with cryptococcalmeningitis diagnosed by India ink staining or cryptococcal antigen (CRAG) test on CSF., were recruited into the study. The exclusion criteria are listed in Table 1.

Table 1

Study exclusion criteria

Any critically ill patient who is unlikely to survive at least three days of the study.

Any patient with contraindication to amphotericin B

Any patient on nephrotoxin known to exacerbate amphotericin B renal toxicity includingacyclovir, aminoglycosides, carboplatin, cisplatin, cyclosporin, foscarnet, frusemide, non-steroidal anti-inflammatory agents, radio-contrast dye, rifampicin, intravenous co-trimoxazole, tacrolimus and vancomycin.

Any patient with uncorrected hypokalaemia at initiation of amphotericin B therapy.

Patients were recruited from the medical wards daily and relevant medical history and physical examination performed by the principal investigator. The patient's general condition was assessed, including the presence of dehydration which was noted and the primary clinician informed on the need for re-hydration. The overall severity of illness was assessed using the Karnofsky performance status scale. Amphotericin B was administered at a dose of $0.7 \mathrm{mg} / \mathrm{kg}$ infused in $500 \mathrm{ml} 5 \%$ dextrose over two to four hours. Potassium was supplemented intravenously as potassium chloride infusion (dose decided by primary clinician). Patients were preloaded with at least a litre of normal saline before each dose of amphotericin B.

Urinalysis (dip-stick method) was done before and at the end or stoppage of the therapy. If negative for protein by dipstick, the sample at end of therapy was assayed for micro-albuminuria. The CD4 count was measured before initiating the therapy. The serum potassium was measured daily for six days then on alternate days thereafter. Serum magnesium and Creatinine were measured at the start of amphotericin B therapy and on days 3, 6, 10 and 14 of therapy. Creatinine clearance $(\mathrm{Ccr})$ estimate was calculated using Cockcroft and Gault formula at the start of therapy and after each serum creatinine measurement. The baseline creatinine clearance at the start of therapy and the lowest creatinine clearance which corresponded with peak creatinine were noted. These two values were analysed to determine any deterioration of renal function.

Changes in creatinine level were also assessed as per the criteria used in other studies $(17,18)$.

Definition 0: Increase in serum creatinine of $<46$ $\mu \mathrm{mol} / \mathrm{L}$ from baseline

Definition 1: Increase in serum creatinine of 46$92 \mu \mathrm{mol} / \mathrm{L}$ from baseline

Definition 2: $50 \%$ increase of serum creatinine over the baseline

Definition 3: Increase in serum creatinine of 93$230 \mu \mathrm{mol} / \mathrm{L}$ from baseline

Definition 4: Doubling in serum creatinine over the baseline

Definition 5: Increase in creatinine to $>230 \mu \mathrm{mol} / \mathrm{L}$

Nephrotoxicity that met any of definitions 1-4 was considered mild to moderate, whereas definition 5 was considered severe. Peak creatinine level was defined as the maximum creatinine value measured for each patient during the study evaluation period. This is the value which was used in analysis. Change in creatinine was the difference between the peak and baseline creatinine.

Hypokalaemia was defined as serum potassium of less than $3.5 \mathrm{mmol} /$ litre, with values of 3-3.49 $\mathrm{mmol} /$ litre classified as significant and less than $3 \mathrm{mmol} /$ litre as severe hypokalaemia. Trough potassium was the lowest potassium value measured during the study period for each patient and this is the value which was analysed. Hypomagnesaemia was defined as serum magnesium less than 0.7 $\mathrm{mmol} / \mathrm{L}$ and the baseline and trough value of the measured serum magnesium were the values analysed.

Data analysis: Data were cleaned and coded before input into SPSS version 12.0.1. The incidence of significant rise in creatinine, hypokalaemia and hypomagnesaemia were calculated. The data were analysed using several different statistical tests to determine univariate associations namely: Pearson correlation, Wilcoxon signed rank test and Fisher's exact test. The results were considered statistically significant at $\mathrm{P}<0.05$. Multiple-regression was applied to determine correlation between variables. 


\section{RESULTS}

Baseline characteristics of the patients studied are shown in Table 2.

Table 2

Baseline characteristics of study population

\begin{tabular}{lcc}
\hline Variable & Frequency & $(\%)$ \\
\hline Gender & 39 & 55.7 \\
Male & 31 & 44.3 \\
Female & & \\
Age group (years) & 2 & 2.9 \\
$<20$ & 20 & 28.6 \\
$21-30$ & 23 & 31.9 \\
$31-40$ & 14 & 20.0 \\
$41-50$ & 11 & 15.7 \\
$>50$ & & \\
Mean age 37.1 \pm 11.13 (range 18-62) & & \\
Basis of diagnosis cryptococcal meningitis & 60 & 85.7 \\
Positive CSF India ink & 6 & 8.6 \\
Positive CSF CRAG & 4 & 5.7 \\
Both India ink and CSF CRAG & & \\
Severity of illness KS Score & 1 & 1.4 \\
$40 \%$ & 2 & 2.9 \\
$60 \%$ & 6 & 8.6 \\
$70 \%$ & 11 & 15.7 \\
$80 \%$ & 19 & 27.1 \\
$90 \%$ & 31 & 44.3 \\
$100 \%$ & & \\
CD4 Count (cell/microlitre) & 47 & 67.1 \\
$<50$ & 16 & 22.9 \\
$50-100$ & 7 & \\
$>100$ & &
\end{tabular}

The results of the mean baseline and mean peak results for baseline and trough serum potassium creatinine values and corresponding estimated creatinine clearance are tabulated in Table 3. Similar and magnesium levels are also shown. All these parameters showed significant changes. 
Table 3

Baseline and peak creatinine and baseline and trough potassium and magnesium

\begin{tabular}{|c|c|c|c|c|c|}
\hline & Mean baseline & Mean peak & Mean trough & Change & $\begin{array}{l}\text { Significance } \\
\text { of change }\end{array}$ \\
\hline Creatinine $(\mu \mathrm{mol} / 1)$ & $75.6^{*}(70.5-80.7)$ & $176.2(158.7-193.8)$ & & 100.6 & $0.000 \quad \mathrm{P}<0.01$ \\
\hline $\begin{array}{l}\text { Creatinine clearance } \\
\left(\mathrm{ml} / \mathrm{min} / 1.73 \mathrm{~m}^{2}\right)\end{array}$ & $93.6(85.8-101.5)$ & & $43.6(38.8-48.4)$ & 50 & $0.000 \quad \mathrm{P}<0.01$ \\
\hline Potassium $(\mathrm{mmol} / \mathrm{l})$ & $4(3.9-4.1)$ & & $2.8(1.9-3.7)$ & 1.2 & $0.000 \mathrm{P}<0.01$ \\
\hline Magnesium (mmol/l) & $0.9(0.85-0.95)$ & & $0.55(0.50-0.59)$ & 0.36 & $0.000 \quad \mathrm{P}<0.01$ \\
\hline
\end{tabular}

${ }^{*}$ Data presented as mean (95\% Confidence Interval)

The severity of nephrotoxicity is shown in Table 4.

Table 4

Classification of nephrotoxicity

\begin{tabular}{llll}
\hline Absolute values & & Percentage rise in creatinine & \\
\hline Nil (def: 0) & $1.5(21.4 \%)$ & Nil $(<50 \%)$ & $24.3 \%$ \\
Mild to moderate (def: $1-4)$ & $51(72.9 \%)$ & $50 \%$ rise serum creatinine (def 2$)$ & $27.1 \%$ \\
Severe & $4(5.7 \%)$ & $100 \%$ rise serum creatinine (def 4$)$ & $58.6 \%$ \\
\hline
\end{tabular}

def $=$ definition

definition of nephrotoxicity is as outlined under materials and methods

Classification by severity of hypokalaemia revealed that $7.1 \%$ of study patientshadnohypokalaemia, $21.7 \%$ had significant (3.0-3.49 mmol/L) hypokalaemia and
$65 \%$ had severe (less than $3 \mathrm{mmol} / \mathrm{L}$ ) hypokalaemia. Correlation of nephrotoxicity, hypokalaemia and hypomagnesaemia with socio-demographic and other variables are as tabulated in Table 5.

Table 5

Correlation of nephrotoxicity, hypokalaemia and hypomagnesaemia with different variables

\begin{tabular}{lllll}
\hline Variable & $\begin{array}{l}\text { Rise in creatinine } \\
\text { level }\end{array}$ & $\begin{array}{l}\text { Fall in creatinine } \\
\text { clearance }\end{array}$ & $\begin{array}{l}\text { Fall in } \\
\text { potassium }\end{array}$ & $\begin{array}{l}\text { Fall in magnesium } \\
\text { level }\end{array}$ \\
\hline Baseline value & $0.000^{* * *}$ & $0.000^{* * *}$ & $0.000^{* * *}$ & $0.000^{* * *}$ \\
KS Score $(\%)$ & 0.561 & 0.069 & 0.368 & 0.760 \\
CD4 count & 0.319 & 0.323 & 0.363 & 0.927 \\
Age group $(<35$ & $0.005^{* *}$ & $0.006^{* *}$ & 0.111 & 0.857 \\
and $>35$ years $)$ & & & & 0.317 \\
Gender/Sex & 0.127 & 0.232 & 0.257 & 0.317 \\
\hline
\end{tabular}

${ }^{* * *} \mathrm{P}<0.001,{ }^{* *} \mathrm{P}<0.01$. All comparisons by Wilcoxon-rank test except by Fisher's exact test 


\section{DISCUSSION}

Therearetwo previouslyused criteria for amphotericin $B$ nephrotoxicity using serum Creatinine levels namely; doubling of creatinine over baseline and a $50 \%$ increase in the baseline serum creatinine and achieving a peak of $2.0 \mathrm{mg} / \mathrm{dl}(177 \mu \mathrm{mol} / \mathrm{L})$.

Using the first definition, the incidence of nephrotoxicity in our study was $58.6 \%$. This is higher than in previous studies, where $24-53 \%$ of the patients studied had nephrotoxicity $(9,19-26)$. Using the second definition, our incidence of nephrotoxicity was $38.6 \%$. This was also higher compared with previous studies where the incidence was $8.5-30 \%$ $(12,27-30)$. The incidence of nephrotoxicity, using any of the two criteria was higher in our study compared to most other studies and this finding is contrary to the expectation as factors which enhance toxicity like concomitantnephrotoxic drugs and dehydration were addressed in the exclusion criteria of our study and not in some of the other studies. Our study also used lower daily and cumulative doses than most of the other studies. A plausible explanation is that AIDS predisposes the patient to greater nephrotoxicity than other underlying conditions (e.g. malignancy) in the other studies. This explanation was also favoured by Bates et al (10) who scored HIV highly as per the Charlson score used to assess severity of illness in their study. Charlson score correlated well with nephrotoxicity.

Another possibility is that the toxicity is higher in the African race which was our study population compared with the Caucasians (majority in the other studies). It is worth noting that there were differences in design (some retrospective) and study population between our study and these other previous studies.

Concerning the classification of severity of nephrotoxicity, the patients in this study had more severe toxicity than in the study by Luber et al (18).

The incidence of hypokalaemia (92.8\%) in our study was much higher than in the previous studies where the incidence ranged between 0 to $83 \%$ with most studies showing an incidence below 20\% (17,31-36).

This is also contrary to expectation considering that all the patients had potassium supplementation, the supplemented amount being decided upon by the primary clinician. A possible explanation is that the supplementation of potassium was inadequate. A second plausible explanation is that the potassium wasting in AIDS patients with cryptococcal meningitis may be more severe than in other populations studied may be due to more severe tubular dysfunction in AIDS.

Thestudy designs and study populations in these other studies were also different from our study.
When further analysed by severity, not only did more of our patients develop hypokalaemia but larger proportion (68\% vs. $30 \%$ ) developed severe hypokalaemia compared with the findings of Bates et al (10). This may be again due to inadequate replacement of potassium or more tubular toxicity in our study population.

Though not an objective of the study, the finding of baseline hypomagnesaemia in $20 \%$ of the study population is interesting and should probably be explored in future studies. The prevalence of hypomagnesaemia $(80 \%)$ was comparable if not higher than in study by Bates et al (10) where the prevalence was $72 \%$. Whang et al (37) suggested that it is difficult to correct hypokalaemia unless one also corrects the hypomagnesaemia. Our study did not correlate hypomagnesaemia with hypokalaemia but our finding of high incidence of hypokalaemia may be partly due to the high prevalence of hypomagnesaemia.

The peak creatinine showed correlation with baseline creatinine and similarly baseline creatinine clearance showed a correlation with creatinine clearance. There was also a significant correlation between peak creatinine (and creatinine clearance derived from the same) and age. This is similar to the findings in the study by Bates et al (10) Therefore, age seems to be a consistent predictor of amphotericin B induced nephrotoxicity.

The other variables (gender, CD4 count, severity of illness) did not show a statistically significant correlation with the peak creatinine. This contrasts with the findings of Bates et al (10) where there was demonstrable significant correlation between severity of illness and nephrotoxicity. A likely explanation for the lack of correlation with severity of illness is that the Karnofsky score as a marker of severity of illness may not correlate with nephrotoxicity unlike the Charlson score. It is imperative to note that AIDS has a high score in the Charlson score and thus the whole of our study population would have a high score for severity of illness and cannot be stratified by the Charlson score. It is also possible that this study had fewer subjects and was not powered to show such correlation especially considering that the results showed a trend despite not being statistically significant.

In conclusion, there is a high incidence of nephrotoxicity, hypokalaemia and hypomagnesaemia in AIDS patients treated for cryptococcal meningitis using amphotericin B deoxycholate at the Kenyatta National Hospital despite efforts aimed at prevention and correction.

The limitations of the study included the fact thathypokalaemia and hypomagnesaemia could have 
been confounded by other factors such as poor feeding and vomiting. Although this study's exclusion criteria took care of the previously studied nephrotoxic drugs, it did not eliminate the other co-administered drugs that may still be nephrotoxic albeit slightly.

\section{REFERENCES}

1. UNAID/WHO. Global summary of HIV/AIDS epidemic. Special report on HIV prevention. December 2007 update; \{online\}. 2007; Available from URL: http://www. unaid. org/ epi/2007/ doc/EPIupdate. $2005-p d f$.

2. Guidelines for ARV Drug Therapy in Kenya. 3rd Edition. Chapter 1, p13. Ministry of health, Republic of Kenya, 2005.

3. French, N.A.B., Gray, K.B., Watera, C.B., et al. Cryptococcal infection in a cohort of HIV-1-infected Ugandan adults. AIDS. 2002; 16: 1031-1038.

4. Jowi, J. O., Mativo, P.M. and Musoke, S. S. Clinical and laboratory characteristics of hospitalized patients with neurological manifestations of HIV / AIDS at the Nairobi Hospital. East Afr. Med. J. 2007; 84: 67-76.

5. Bicanic, T. and Harrison, T.S. Cryptococcal meningitis. Brit. Med. Bull. 2005; 18: 99-118.

6. Branch, R.A. Prevention of Amphotericin B-induced renal impairment. A review on the use of sodium supplementation. Arch. Intern. Med. 1988; 148:23892394.

7. Sawaya, B.P., Briggs, J.P. and Schnermann, J. Amphotericin B nephrotoxicity: The adverse consequences of altered membrane properties. J. Amer. Soc. Nephrol. 1995; 6:154-164.

8. Butler, W.T., Bennett, J.E., Alling D.W., et al. Nephrotoxicity of Amphotericin B; early and late effects in 81 patients. Ann. Intern. Med. 1964; 61:175187.

9. Harbarth, S., Pestotnik, S.L., Lloyd, J.F., et al. The epidemiology of nephrotoxicity associated with conventional Amphotericin B therapy. Amer. J. Med. 2001; 111: 528-534.

10. Bates, D.W., Su, L., Yu, D.T., et al. Correlates of acute renal failure in patients receiving parenteral Amphotericin B. Kidney Inter. 2001; 60: 1452-1459.

11. Barton, C.H., Pohl, M., Vaziri, N.D. and Cesario, T. Renal magnesium wasting associated with Amphotericin B therapy. Amer. J. Med. 1984; 77:471-474.

12. Harbarth, S., Burke, J.P., Lloyd, J.F., et al. Clinical and economic outcomes of conventional Amphotericin B-associated nephrotoxicity. Clin. Infect. Dis. 2002; 35: $120-127$.

13. Ullmann, A.J., Sanz, M.A, Tramarin, A., et al. Prospective study of Amphotericin B formulations in immunocompromised patients in 4 European countries. Clin. Infect. Dis. 2006; 43: 29-38.

14. Susan, H. Amphotericin B-related nephrotoxicity increases hospital stay in immunocompromised patients. Cancer weekly, September 26, 2006.

15. Heidemann, H.T., Gerkens, J.F., Spickard, W.A., et al. Amphotericin B nephrotoxicity in humans decreased by salt repletion. Amer. J. Med. 1983; 75: 476-483.
16. Sacks, P. and Fellner, S.K. Recurrent reversible acute renal failure from Amphotericin B. Arch. Intern. Med. 1987; 147:593-595.

17. Clements, J. S. and Peacock J. E. Amphotericin B revisited: reassessment of toxicity. Amer. J. Med. 1990. 88 Suppl. $5 \mathrm{~N}$ : 22N-27N.

18. Luber, A.D., Maa, L., Lam, M. and GuglielmoJ. B. Risk factors for Amphotericin B induced nephrotoxicity. J. Antimicrob. Chemother.1999; 43: 267-271.

19. Benson, J.M. and Nahata, M.C. Pharmacokinetics of Amphotericin B in children. Antimicrob. Agents Chemother. 1989; 33: 1989-1993.

20. Walsh, T.J., Finberg, RW., Arndt, C., et al. Liposomal Amphotericin B for empirical therapy in patients with persistent fever and neutropenia National Institute of Allergy and Infectious Diseases Mycoses Study Group. N. Engl. J. Med. 1999; 340:764-771.

21. Wingard, J.R., Kubilis, P., Lee, L., et al. Clinical significance of nephrotoxicity in patients treated with amphotericin B for suspected or proven aspergillosis. Clin. Infect. Dis. 1999; 29:1402-1407.

22. Prentice, H.G., Harm, L.M., Herbrecht, R., et al. A randomized comparison of liposomal versus conventional amphotericin B for the treatment of pyrexia of unknown origin in neutropenic patients. Br. J. Haematol. 1997; 98:711-718.

23. White, M.H., Bowden, R.A., Sandler, E., et al. Randomized, double-blind clinical trial of amphotericin B colloidal dispersion vs. amphotericin $B$ in the empirical treatment of fever and neutropenia. Clin. Infect. Dis. 1998; 27:296-302.

24. Herbrecht, R., Denning, D.W., Patterson, T.F., et al. Voriconazole versus amphotericin B for primary therapy of invasive aspergillosis. N. Engl. J. Med. 2002; 347: 408-415.

25. Mora-Duarte, J., Betts, R., Rotstein, C., et al. Comparison of caspofungin and amphotericin B for invasive candidiasis. N. Engl. J. Med. 2002; 347: 2020-2029.

26. Kullberg, B.J., Sobel, J.D., Ruhnke, M., et al. Voriconazole versus a regimen of amphotericin B followed by fluconazole for candidaemia in nonneutropenic patients: a randomised non-inferiority trial. Lancet. 2005; 366: 1435-1442.

27. Bates, D.W., Su, L., Yu, D.T., et al. Mortality and cost of acute renal failure associated with amphotericin B therapy. Clin. Infect. Dis. 2001; 32:686-693.

28. Chien-Yuan, Chen, Ritesh, N., Kumar, Yin-Hsun Feng, et al. Treatment outcomes in patients receiving conventional amphotericin B therapy: a prospective multicentre study in Taiwan. J. Antimicrob. Chemother. 2006; 57: 1181-1188.

29. Girmenia, C, Gentile, G., Micozzi, A., et al. Nephrotoxicity of amphotericin B desoxycholate. Clin. Infect. Dis. 2001; 33: 915-916.

30. Girmenia, C., Cimino, G., Micozzi, A., et al. Risk factors for nephrotoxicity associated with conventional amphotericin B therapy. Am. J. Med. 2002; 113:351.

31. Pathak, A., Pien, F. D. and Carvalho, L. Amphotericin B use in a community hospital, with special emphasis on side effects. Clin. J. Infect. Dis. 1998; 26:334 -338. 
32. Sanford, W. G., Rasch, J. R. and Stonehill, R. B. A therapeutic dilemma: the treatment of disseminated coccidioidomycosis with amphotericin B. Ann. Intern. Med. 1962; 56: 553-563.

33. Miller, R.P. and Bates, J.R. Amphotericin B toxicity: a follow-up report of 53 patients. Ann. Intern. Med. 1969; 71: 1089-1095.

34. Medoff, G., Dismukes, W.E., Meade, R.H. III and Moses, J.M. A new therapeutic approach to Candida infections: a preliminary report. Arch. Intern. Med. 1972; 130: 241-245.
35. Gross, M.H., Pickard, W.W. and Perfect, J.R. Retrospective review of amphotericin use in a tertiarycare medical center. Am. J. Hosp. Pharm. 1987; 44: 1353-1357.

36. Anaissie, E.J., Vartivarian, S.E., Abi-Said, D., et al. Fluconazole vs. amphotericin B in the treatment of hematogenous candidiasis: a matched cohort study. Am. J. Med. 1996; 101: 170-176.

37. Whang, R., Whang, D.D. and Ryan, M.P. Refractory potassium depletion. A consequence of magnesium deficiency. Arch. Intern. Med. 1992; 152:40-45. 